\title{
Cytogenetic findings in lung cancer that illuminate its biological history from adenomatous hyperplasia to bronchioalveolar carcinoma to adenocarcinoma: A case report
}

\author{
DANIELA BETTIO ${ }^{1}$, UMBERTO CARIBONI ${ }^{2}$, ANNA VENCI $^{1}$, MARIALUISA VALENTE ${ }^{1}$, \\ PAOLA SPAGGIARI $^{3}$ and MARCO ALLOISIO ${ }^{2}$ \\ ${ }^{1}$ Cytogenetic and Medical Genetic Laboratory, Operative Unit of Clinical Investigations; \\ Departments of ${ }^{2}$ Thoracic Surgery and ${ }^{3}$ Pathology, IRCCS Humanitas Clinical Institute, \\ Rozzano, Milan, Italy
}

Received June 11, 2012; Accepted August 21, 2012

DOI: $10.3892 /$ etm.2012.725

\begin{abstract}
The biological and chronological evolution of lung cancer remain to be fully elucidated. A multi-step carcinogenesis hypothesis suggests a progression from atypical adenomatous hyperplasia (AAH) through bronchioalveolar carcinoma (BAC) to invasive adenocarcinoma (AC), but to date this has not been formally demonstrated. We report a case of a patient diagnosed by computed tomography (CT) with lung cancer in the superior right lobe who also presented with a pure ground-glass opacity (GGO) in the inferior lobe, while the middle lobe appeared normal. Following pneumonectomy, cytogenetic analysis successfully performed on spontaneous metaphases obtained by the direct method from samples of the three lung lobes showed the presence of three clonal cell populations, each progressively having increased karyotype complexity. Fluorescence in situ hybridization (FISH), performed using $A L K(2 \mathrm{p} 23)$ break probe and $A L K /$ $E M L 4 \mathrm{t}(2 ; 2) ; \operatorname{inv}(2)$ fusion probe, showed a normal pattern for all specimens. Histological evaluation confirmed the presence of $\mathrm{AC}$ in the superior right lobe and classified the GGO lesion as BAC and the normal tissue of the middle lobe as AAH. To the best of our knowledge, this is the first case in which the cytogenetic study of spontaneous metaphases showed a clear clonal relationship among $\mathrm{AC}, \mathrm{BAC}$ and $\mathrm{AAH}$ present simultaneously in different lobes of the same lung. This case appears to indicate that the entire lung was somehow predisposed to a neoplastic transformation starting with a diffuse AAH characterized by high proliferative activity. Moreover,
\end{abstract}

Correspondence to: Dr Daniela Bettio, Cytogenetic Laboratory, IRCCS Humanitas Clinical Institute, Via Manzoni 56, I-20089 Rozzano, Milan, Italy

E-mail: daniela.bettio@humanitas.it

Key words: lung cancer, ground-glass opacities, chromosome abnormalities, multi-step progression the $5 \mathrm{q} 13$ region involved in the translocation shared by BAC and $\mathrm{AC}$ contains at least 4 genes encoding important regulators of the cell cycle that may be considered new molecular markers of lung cancer.

\section{Introduction}

The pathogenesis of lung cancer and the criteria that regulate its progression are under investigation. Pulmonary lesions, such as small nodules with focal ground-glass opacity (GGO), have been increasingly detected due to the widespread use of computed tomography (CT) scanning. Histologically, these lesions can be classified as atypical adenomatous hyperplasia (AAH), bronchioalveolar carcinoma (BAC) or adenocarcinoma (AC). Several studies have suggested that AAH, frequently found in tissue surrounding lung $\mathrm{AC}$, may be a forerunner in the development of AC; moreover, the more recent discovery of lung nodules manifesting as GGOs further supports a stepwise process in the development of pulmonary AC (1-3). However, the genetic relationship between $\mathrm{AC}$ and the associated foci of AAH is not yet well defined. In particular, it is not clear whether multiple foci of $\mathrm{AAH}$ and $\mathrm{AC}$ in the same patients are clonally related or are independent neoplastic foci (4). Several studies performed loss of heterozygosity $(\mathrm{LOH})$, fluorescence in situ hybridization (FISH), microarrays and immunohistochemistry analyses and demonstrated an increasing genetic complexity associated with lung cancer progression (4-7) but, to the best of our knowledge, no cytogenetic study showing a clear clonal relationship among AC, BAC and AAH has been reported thus far.

We report the case of a patient histologically diagnosed with $\mathrm{AC}$ in the superior right lobe and $\mathrm{BAC}$ in the inferior lobe, previously identified as a pure GGO nodule by a CT scan. AAH was diagnosed in the middle lobe, considered to be normal at CT scan and during surgery. The cytogenetic studies performed on biopsies from the three lobes allowed the identification of different chromosome rearrangements with clonal evolution that supports the hypothesis of a complex multi-step carcinogenesis in which lung AC develops from AAH through BAC. 


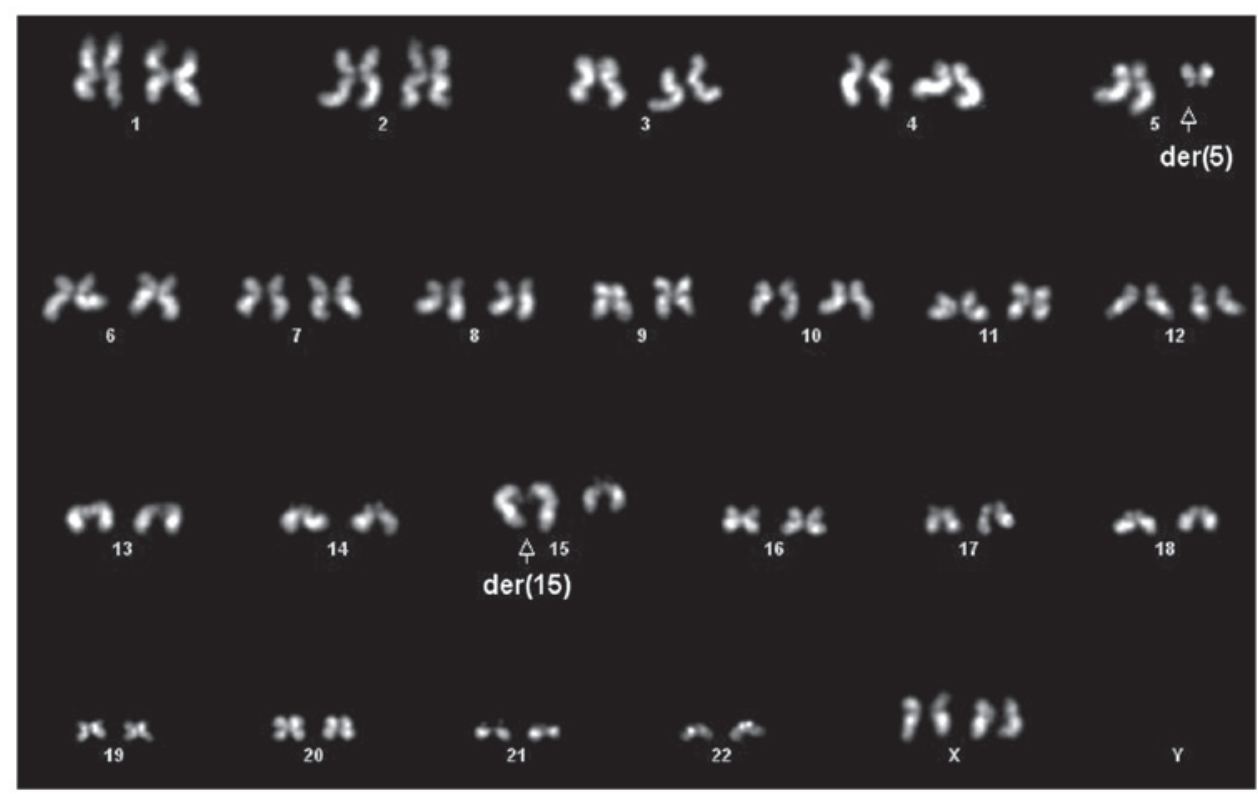

Figure 1. 46,XX,t(5;15)(q13;q25-26) karyotype observed in the ground glass opacity lesion.

\section{Case report}

A 54-year-old female who never smoked was diagnosed with a lung tumor in the upper right lobe by a CT scan. A transthoracic fine-needle aspiration of the lesion was performed, followed by histological diagnosis of $\mathrm{AC}$ with a BAC component. The $\mathrm{CT}$ scan also showed a pure GGO lesion in the lower lobe, while the middle lobe appeared to be normal. The patient underwent pneumonectomy and samples from the 3 lung lobes were sent to the cytogenetic laboratory. Cytogenetic analyses were performed on spontaneous metaphases obtained by the direct method and short-term cultures as previously reported (8). The normal appearing middle lobe showed high spontaneous replication activity after $24 \mathrm{~h}$ of incubation, with 9 of 9 cells presenting a normal karyotype. The GGO lesion had 4 cells with a $\mathrm{t}(5 ; 15)(\mathrm{q} 13 ; \mathrm{q} 25-26)$ as a single anomaly (Fig. 1), 2 cells with the $\mathrm{t}(5 ; 15)$ translocation with complex rearrangements, including a derivative chromosome 1 with unknown additional material on the short arm, and 4 cells with a normal karyotype. In the $\mathrm{AC}$, the same rearrangements present in the GGO were observed in 9 of 9 metaphases, 2 of which included the $t(5 ; 15)$. Most of the metaphases showing complex rearrangements were incomplete and in these cases we defined a composite karyotype following the ISCN recommendations based on the recurrent abnormalities observed (9): 44 46,X,del(X) (p11.2),der(1)add(1)(p32),t(5;15)(q13;q25-26) [cp9].

The karyotypes obtained from all samples after short-term cultures (5-7 days) were normal, indicating that the tumor cytogenetic profile is rapidly obscured in short-term cultures due to a selective advantage of karyotypically normal cells, as previously reported (8).

Since a fusion gene between echinoderm microtubuleassociated protein-like 4 (EML4) and anaplastic lymphoma kinase $(A L K)$ has been identified in a subset of non-small cell lung cancer patients who never smoked (10), FISH was performed on our specimens using the $A L K(2 \mathrm{p} 23)$ break probe and $A L K / E M L 4 \mathrm{t}(2 ; 2)$; inv(2) Fusion Probe (Poseidon ${ }^{\mathrm{TM}}$, a gift from Kreatech Diagnostics, The Netherlands). A normal pattern was observed. This case report was part of a research project approved by the local ethics committee (authorization No. 850), and informed patient consent was obtained.

\section{Discussion}

The molecular drivers that determine histology in lung cancer remain largely unknown and it is difficult to identify a valid parameter of tumor aggressiveness that may be used as a prognostic factor. The hypothesis of a multi-step carcinogenic process has recently been supported by the observations of Min et al (11) in a patient over a 10-year follow-up period, in whom CT and PET imaging findings showed the progression from a focal pure GGO nodule (presumed to be AAH or BAC) to an invasive $\mathrm{AC}$.

The cytogenetic findings in our case support this hypothesis. The observation of active replication with spontaneous metaphases obtained after a few hours of incubation in the biopsy from the middle lobe suggests that in the normal appearing tissue the cell cycle control was lost. It is well known that cancer is a disease of hyper-proliferation predisposing to chromosome instability and this may have led to the first rearrangement we identified in the sample from the GGO, the translocation $t(5 ; 15)(q 13 ; q 25-26)$. Notably, the breakpoint at band q13 in the long arm of chromosome 5 is the same as we observed in a constitutional pericentric inversion previously reported in a patient with a pure GGO lesion (12). This band contains at least 4 genes (CCNB1, CDK7, CENPH, RAD17) encoding important regulators of the cell cycle that could be disrupted by the chromosome rearrangement (13). Moreover, a genome-wide association study reported that the chromosome $15 q 25.1$ region, which includes three nicotinic cholinergic receptor genes (CHRNA5, CHRNB4, CHRN) and cell proliferation gene (PSMA4), is associated with lung 
cancer risk in Caucasian individuals irrespective of smoking status or propensity to smoke tobacco (14). In our case, the clone with the complex karyotype which was present in a few cells from the GGO and in all the cells from the AC probably had a strong proliferative advantage on the clones harboring the $t(5 ; 15)$. This is in agreement with the well-known observation that the complexity of chromosomal aberrations in cancer is correlated with the aggressiveness of the disease.

Common gene variants involved in lung cancer have been recently identified through large, collaborative, genome-wide association studies. Three loci markedly associated with lung cancer susceptibility have been reported: 5p15, 6p21 and 15q25, where genes that regulate acetylcholine niconitic receptors and telomerase production are located (15). In the present case, two of these three relevant regions were involved in chromosome rearrangements that may either cause gene inactivation or dysregulation, supporting their crucial role in the disease.

To the best of our knowledge, this is the first study to report a clonal relationship among AC, BAC and AAH, present simultaneously in different lobes of the same lung. This case suggests that the entire lung was somehow prone to the neoplastic transformation, possibly primed by cells with high proliferative activity such as those present in the middle lobe affected by AAH. Genetic studies of multiple lesions present in the same lung should be performed in order to verify this hypothesis.

\section{Acknowledgements}

The authors wish to thank Dr Robert Nicholls for the critical reading of the manuscript.

\section{References}

1. Kitamura H, Kameda Y, Ito T and Hayashi H: Atypical adenomatous hyperplasia of the lung. Implications for the pathogenesis of peripheral lung adenocarcinoma. Am J Clin Pathol 111: 610-622, 1999.

2. Henschke CI, Yankelevitz DF, Mirtcheva R, McGuinness G, McCauley D and Miettinen OS; ELCAP Group: CT screening for lung cancer: frequency and significance of part-solid and nonsolid nodules. AJR Am J Roentgenol 178: 1053-1057, 2002.

3. Nakata M, Saeki H, Takata I, Segawa Y, Mogami H, Mandai K and Eguchi K: Focal ground-glass opacity detected by low-dose helical CT. Chest 121: 1464-1467, 2002.
4. Morandi L, Asioli S, Cavazza A, Pession A and Damiani S: Genetic relationship among atypical adenomatous hyperplasia, bronchioloalveolar carcinoma and adenocarcinoma of the lung. Lung Cancer 56: 35-42, 2007.

5. Kerr KM, Carey FA, King G and Lamb D: Atypical alveolar hyperplasia: relationship with pulmonary adenocarcinoma, p53, and c-erbB-2 expression. J Pathol 174: 249-256, 1994.

6. Tomida S, Yatabe Y, Yanagisawa K, Mitsudomi T and Takahashi T: Throwing new light on lung cancer pathogenesis: updates on three recent topics. Cancer Sci 96: 63-68, 2005.

7. Sano T, Kitayama Y, Igarashi H, Suzuki M, Tanioka F, Chida K, Okudela K and Sugimura H: Chromosomal numerical abnormalities in early stage lung adenocarcinoma. Pathol Int 56: 117-125, 2006.

8. Bettio D, Rizzi N, Giardino D, Persani L, Pecori-Giraldi F, Losa M and Larizza L: Cytogenetic study of pituitary adenomas. Cancer Genet Cytogenet 98: 131-136, 1997.

9. ISCN 2009: An international system for human cytogenetic nomenclature. Shaffer LG, Slovak ML, Campbell LJ (eds). Karger, Basel, pp1-138, 2009.

10. Mano H: Non-solid oncogenes in solid tumors: EML4-ALK fusion genes in lung cancer. Cancer Sci 99: 2349-2355, 2008.

11. Min JH, Lee HY, Lee KS, Han J, Park K, Ahn MJ and Lee SJ: Stepwise evolution from a focal pure pulmonary ground-glass opacity nodule into an invasive lung adenocarcinoma: An observation for more than 10 years. Lung Cancer 69: 123-126, 2010.

12. Bettio D, Venci A, Cariboni U, Di Rocco M and Infante $\mathrm{M}$ : Fluorescent in situ hybridization (FISH) in the differential diagnosis of ground-glass opacities in the lung. Lung Cancer 71: 319-322, 2011.

13. UCSC Genome Browser (database online). Santa Cruz: University of California, Genome Bioinformatics Group; 2003. Updated February, 2009. Available at: http://genome.ucsc.edu/ cgi-bin/hgGateway. Accessed September 22, 2011.

14. Hung RJ, McKay JD, Gaborieau V, Boffetta P, Hashibe M, Zaridze D, Mukeria A, Szeszenia-Dabrowska N, Lissowska J, Rudnai P, Fabianova E, Mates D, Bencko V, Foretova L, Janout V, Chen C, Goodman G, Field JK, Liloglou T, Xinarianos G, Cassidy A, McLaughlin J, Liu G, Narod S, Krokan HE, Skorpen F, Elvestad MB, Hveem K, Vatten L, Linseisen J, Clavel-Chapelon F, Vineis P, Bueno-de-Mesquita HB, Lund E, Martinez C, Bingham S, Rasmuson T, Hainaut P, Riboli E, Ahrens W, Benhamou S, Lagiou P, Trichopoulos D, Holcátová I, Merletti F, Kjaerheim K, Agudo A, Macfarlane G, Talamini R, Simonato L, Lowry R, Conway DI, Znaor A, Healy C, Zelenika D, Boland A, Delepine M, Foglio M, Lechner D, Matsuda F, Blanche H, Gut I, Heath S, Lathrop M and Brennan P: A susceptibility locus for lung cancer maps to nicotinic acetylcholine receptor subunit genes on 15q25. Nature 452: 633-637, 2008.

15. Brennan P, Hainaut P and Boffetta P: Genetics of lung-cancer susceptibility. Lancet Oncol 12: 399-408, 2011. 